\title{
Regularized estimation of Euler pole parameters
}

\author{
Bahadır Aktuğ $^{1}$ and Ömer Yıldırım² \\ ${ }^{1}$ Geodesy Department, Kandilli Observatory and Earthquake Research Institute, Bogazici University, 34684 Cengelkoy, Istanbul, Turkey \\ ${ }^{2}$ Department of Surveying Engineering, Gaziosmanpaşa University, 60250 Tokat, Turkey
}

(Received December 5, 2011; Revised September 29, 2012; Accepted October 20, 2012; Online published August 23, 2013)

\begin{abstract}
Euler vectors provide a unified framework to quantify the relative or absolute motions of tectonic plates through various geodetic and geophysical observations. With the advent of space geodesy, Euler parameters of several relatively small plates have been determined through the velocities derived from the space geodesy observations. However, the available data are usually insufficient in number and quality to estimate both the Euler vector components and the Euler pole parameters reliably. Since Euler vectors are defined globally in an Earth-centered Cartesian frame, estimation with the limited geographic coverage of the local/regional geodetic networks usually results in highly correlated vector components. In the case of estimating the Euler pole parameters directly, the situation is even worse, and the position of the Euler pole is nearly collinear with the magnitude of the rotation rate. In this study, a new method, which consists of an analytical derivation of the covariance matrix of the Euler vector in an ideal network configuration, is introduced and a regularized estimation method specifically tailored for estimating the Euler vector is presented. The results show that the proposed method outperforms the least squares estimation in terms of the mean squared error.
\end{abstract}

Key words: Tectonics, Euler parameters, multicollinearity, GNSS velocities.

\section{Introduction}

The motion of tectonic plates is usually parameterized on a sphere through an Euler vector or an Euler pole (DeMets et al., 1990; Altamimi et al., 2002; Sella et al., 2002). Such a parameterization is also useful to compare the estimates from different sources such as space geodetic measurements, hot-spots tracks, transform fault azimuths, the spreading rates of ocean ridges, and earthquake slip vectors (Gripp and Gordon, 1990, 2002; Argus and Gordon, 1991; DeMets et al., 1994, 2010). However, all the nongeodetic methods give, in fact, only a relative measure of the plate motions. Even the direct geodetic measurements of plate motions depend on the underlying reference frame (Altamimi et al., 2002; Kreemer et al., 2003; Prawirodirdjo and Bock, 2004). Since only the relative motion of the plates can be directly observed, they are often referenced with respect to either a specific plate or a global plate circuit, called no-net-rotation. Thus, the Euler vectors are necessary to compute the global plate circuit closure and to quantify the relative motions of tectonic plates.

The Euler parameterization of tectonic plate motions provides an indispensible tool for modeling the rigid plates where the deformation along the plate boundaries are neglected or assumed comparatively small (McClusky et al., 2000; Nocquet et al., 2001; Aktuğ et al., 2009a, b). Even in those models which take the deformation along plate boundaries into consideration, the Euler parameterization is still employed with the compensation of the deformation

Copyright (c) The Society of Geomagnetism and Earth, Planetary and Space Sciences (SGEPSS); The Seismological Society of Japan; The Volcanological Society of Japan; The Geodetic Society of Japan; The Japanese Society for Planetary Sciences; TERRAPUB.

doi:10.5047/eps.2012.10.004 along plate boundaries through elastic back-slip modeling (McCaffrey, 1996, 2002; Meade and Hager, 2005). In this respect, a reliable estimation of the Euler vector is important in many studies ranging from the estimation of slips along plate boundaries and paleomagnetic studies to the reconstruction of the plate tectonics. It is common to estimate an Euler vector for a plate motion through the velocity vectors obtained from the geodetic measurements. On the other hand, since quantifying the motion of tectonic plates requires a rigidity assumption, the geometrical coverage of the available velocity vectors is limited to the rigid parts of the plate in question. This results in a weakly multicollinear estimation problem, especially for smaller plates. Such multicollinearity can be observed in the high correlation between the Euler vector components, as well as between the Euler pole and its angular velocity. The correlations can be to such an extent that the Euler pole position and its angular rotation rate cannot be estimated directly. This is chiefly due to the matrix singularity of the normal equation matrix, which arises from the collinearity of the rotation rate with either latitude or longitude of the Euler pole. One common solution is to estimate the Euler vector and then transform it to the Euler pole parameters. However, the correlations between the Euler vector components are still close to unity, which still presents a weakly multicollinear problem. The multicollinearity in the estimation of Euler vectors is often coupled with errors which come from the non-rigid behavior of the sites. Up to 3-4 $\mathrm{mm} / \mathrm{yr}$ residuals are common after removing the Euler rotation from the site velocities (Qiang et al., 1999; Nocquet et al., 2001; Aktuğ et al., 2009b). The multicollinearity in the estimation problems is often handled with TykhonovPhillips regularization (Tykhonov, 1963; Phillips, 1962). 
While the required Tykhonov matrix is obvious in many applications, due to geometrical or physical relations such as the smoothness of the geophysical signal over a spatial or temporal domain, the components of the Euler vector are discrete and no such simple relations between parameters are available for Euler vector components. For instance, no direct assumption can be made about any smoothness or closeness between Euler vector components.

Another common choice for the Tykhonov matrix is the identity matrix. This approach is useful to stabilize a singular problem numerically and leads to a minimum norm solution, but it has no physical or geometrical meaning for Euler vector components. Consequently, the necessary Tykhonov matrix, which can be used in the regularized estimation of the Euler vector, is not available and has to be developed analytically as implemented in this study.

In this study, the concept of an ideal distribution of the velocity vectors is introduced, the necessary Tykhonov matrix was derived analytically, and the multicollinearity between Euler parameters was handled with regularization. The results show that the suggested methodology outperforms the standard least squares in terms of the Mean Squared Error (MSE) and decreases the correlation among the Euler vector components.

\section{Regularized Estimation of the Euler Vector}

Since the derivation of the necessary regularization matrix for the Euler vector and the associated equations will be based on the least-squares, the estimation model will be given very briefly for completeness rather than a complete summary. The estimation of the Euler vector from a set of velocities can be formulated in a Gauss-Markov model as:

$$
\mathbf{e}=\mathbf{A} \boldsymbol{\xi}+\mathbf{W}, \quad \mathbf{e} \sim N\left(0, \Sigma_{e}\right),
$$

where $\mathbf{e}$ is the error vector of the velocities, $\mathbf{A}$ is the matrix of coefficients, $\boldsymbol{\xi}$ is the unknown Euler vector $\left(\dot{\omega}_{x}, \dot{\omega}_{y}, \dot{\omega}_{z}\right)$, and $\mathbf{W}$ is the misclosure vector defined as:

$$
\mathbf{W}=\mathbf{A} \boldsymbol{\xi}_{0}-v
$$

where $\boldsymbol{\xi}_{0}$ is the a priori values vector of the parameters $\left(\dot{\omega}_{x}\right.$, $\left.\dot{\omega}_{y}, \dot{\omega}_{z}\right)$, and $v$ is the observation vector which consists of the Cartesian velocity components. The coefficients matrix $\mathbf{A}$ is a function of the mathematical relation between the observed velocity vector and the Euler vector, and can be expressed in tensor notation as:

$$
v_{k}=\xi_{i} r_{j} \varepsilon_{i j k}
$$

where $\varepsilon_{i j k}$ is the permutation tensor and $r$ is the position vector in Cartesian frame. After rearranging Eq. (3) in a matrix form according to the model in Eq. (1), three rows of the matrix A corresponding to the velocity vector at site $i$ can be obtained as;

$$
v_{i}=\left[\begin{array}{ccc}
0 & z_{i} & -y_{i} \\
-z_{i} & 0 & x_{i} \\
y_{i} & -x_{i} & 0
\end{array}\right]\left[\begin{array}{c}
\dot{\omega}_{x} \\
\dot{\omega}_{y} \\
\dot{\omega}_{z}
\end{array}\right]
$$

The whole coefficient matrix A is formed by stacking (4) for each site. In a standard least squares estimation, the $a$ posteriori covariance of the Euler vector components can be obtained as:

$$
\Sigma_{\hat{\boldsymbol{\xi}}}=\left(\mathbf{A}^{T} \Sigma_{e}^{-1} \mathbf{A}\right)^{-1},
$$

where the subscript $\hat{\xi}$ is the vector of the estimated parameters. Hereafter, in this paper, the hat symbol is used to refer to estimated quantities. Assuming $\Sigma_{e}=\sigma^{2} \mathbf{I}$ for an ideal network configuration, where $\sigma^{2}$ is the variance factor and I is the identity matrix, the inverse cofactor matrix of the parameters in Eq. (5) for $n$ sites can be written in an expanded form as:

$$
\Sigma_{\hat{\xi}}^{-1}=\sigma^{-2}\left[\begin{array}{ccc}
\sum_{i=1}^{n}\left(y_{i}^{2}+z_{i}^{2}\right) & -\sum_{i=1}^{n} x_{i} y_{i} & -\sum_{i=1}^{n} x_{i} z_{i} \\
-\sum_{i=1}^{n} x_{i} y_{i} & \sum_{i=1}^{n}\left(x_{i}^{2}+z_{i}^{2}\right) & -\sum_{i=1}^{n} y_{i} z_{i} \\
-\sum_{i=1}^{n} x_{i} z_{i} & -\sum_{i=1}^{n} y_{i} z_{i} & \sum_{i=1}^{n}\left(x_{i}^{2}+y_{i}^{2}\right)
\end{array}\right] .
$$

A regularized estimation for the model Eq. (1) can be formulated by imposing a linear stochastic constraint equation $\Gamma\left(\boldsymbol{\xi}-\boldsymbol{\xi}_{0}\right)=\mathbf{e}_{\boldsymbol{\Gamma}}$, with $\mathbf{e}_{\boldsymbol{\Gamma}} \sim N\left(0, \kappa^{-2} \mathbf{I}\right)$, on the parameters and the estimates can be obtained as:

$$
\hat{\boldsymbol{\xi}}=\boldsymbol{\xi}_{0}-\left(\mathbf{A}^{T} \Sigma_{e}^{-1} \mathbf{A}+\kappa^{2} \boldsymbol{\Gamma}^{T} \boldsymbol{\Gamma}\right)^{-1} \mathbf{A}^{T} \Sigma_{e}^{-1} \mathbf{W},
$$

where $\Gamma$ is the Tykhonov matrix, $\kappa$ is the regularization constant which controls the relative weight between the weighted sum of the residual squares and a priori constraints, and $\mathbf{W}$ is the misclosure vector given in Eq. (2). The derivation of Eq. (7) can be found in many textbooks of inverse problems (e.g., Tarantola, 2005; Hansen, 2010). Equation (7) can be interpreted in a regularization framework as well as in a Bayesian point of view, and it is also known as the Tykhonov-Phillips regularization (Tykhonov, 1963; Phillips, 1962) or ridge regression (Hoerl and Kennard, 1970). The regularization is a mathematical tool which provides additional constraints on the parameters. In this way, many ill-posed problems can be solved or can be made stable. In particular, when the number of parameters exceeds the number of observations, the regularization is indispensible and makes the ill-posed problem solvable. Many examples can be found in the literature for the application of the regularization, such as determining the slip distribution of fault systems (Aktuğ et al., 2010), ionospheric tomography (Howe et al., 1998), and tropospheric tomography (Flores et al., 2000). The benefit of the regularized estimation is two-fold: (1) to provide a priori external information to strengthen the solution, and (2) to stabilize the solution numerically when the solution is ill-conditioned (Tarantola, 2005). For many problems, the Tykhonov matrix $\Gamma$ is taken as an identity matrix, which is just sufficient to stabilize the solution. In many other geophysical problems, the Tykhonov matrix is obvious due to some physical relations between the parameters, such as the smoothness of a parameter group or their gradients within the neighborhood. For instance, Aktuğ et al. (2010) has employed the Laplacian matrix for the Tykhonov matrix to estimate the slip distribution from coseismic GPS displacements. Similarly, Aktuğ (2012) derived a necessary specific 
Tykhonov matrix to estimate the datum transformation parameters from a set of distorted terrestrial network coordinates. For the case of Euler vector components, an analytical derivation based on the intrinsic geometrical relations between the components of an Euler vector is needed. The regularization can be applied with respect to the inverse cofactor matrix of an ideal network configuration by choosing the quadratic Tykhonov matrix as (Tarantola, 2005):

$$
\Gamma^{T} \Gamma=\Sigma_{\hat{\xi}}^{-1}
$$

Assuming that an ideal regional network contains all the theoretically-possible points within a region bounded by the latitudes $\varphi_{a}$ and $\varphi_{b}$, and longitudes $\lambda_{a}$ and $\lambda_{b}$, the individual summation terms in Eq. (6) can be expressed as surface integrals over the region. Since the region is defined in geographic coordinates, it is more convenient to apply the integration over the geodetic coordinates instead of Cartesian coordinates. For the purpose of the following derivation, a spherical approximation to the transformation between Cartesian and the geodetic coordinate system is sufficient:

$$
\begin{aligned}
& x_{i}=R \cos \varphi_{i} \cos \lambda_{i}, \\
& y_{i}=R \cos \varphi_{i} \sin \lambda_{i}, \\
& z_{i}=R \sin \varphi_{i},
\end{aligned}
$$

where $R$ is the approximate Earth's radius. The individual summation terms in the inverse cofactor matrix in (6) can equivalently be expressed as the product of the number of points and the mean values of the functions, and, thus, can be obtained analytically using the integral mean value theorem. The summation terms in (6) can be written as double integrals over the domain $\Delta \varphi=\varphi_{b}-\varphi_{a}$ and $\Delta \lambda=$ $\lambda_{b}-\lambda_{a}$. Denoting the mean latitude and mean longitude as $\varphi_{m}$ and $\lambda_{m}$, respectively, the individual summation terms for a number of points $(n)$ can be written as:

$$
\begin{aligned}
& -\sum_{i=1}^{n} x_{i} y_{i}=\frac{-n R^{2}}{\Delta \varphi \Delta \lambda} \int_{\varphi_{a}}^{\varphi_{b}} \int_{\lambda_{a}}^{\lambda_{b}} \cos ^{2} \varphi \cos \lambda \sin \lambda d \lambda d \varphi \\
& =\frac{-n R^{2} \sin 2 \lambda_{m} \sin \Delta \lambda}{4 \Delta \varphi \Delta \lambda}\left(\Delta \varphi+\cos 2 \varphi_{m} \sin \Delta \varphi\right), \\
& -\sum_{i=1}^{n} x_{i} z_{i}=\frac{-n R^{2}}{\Delta \varphi \Delta \lambda} \int_{\varphi_{a}}^{\varphi_{b}} \int_{\lambda_{a}}^{\lambda_{b}} \cos \varphi \sin \varphi \cos \lambda d \lambda d \varphi \\
& =\frac{-n R^{2} \cos \lambda_{m} \sin (\Delta \lambda / 2) \cos 2 \varphi_{m} \sin \Delta \varphi}{\Delta \varphi \Delta \lambda}, \\
& -\sum_{i=1}^{n} y_{i} z_{i}=\frac{-n R^{2}}{\Delta \varphi \Delta \lambda} \int_{\varphi_{a}}^{\varphi_{b}} \int_{\lambda_{a}}^{\lambda_{b}} \cos \varphi \sin \varphi \sin \lambda d \lambda d \varphi \\
& =\frac{-n R^{2} \sin \lambda_{m} \sin (\Delta \lambda / 2) \sin \varphi_{m} \sin (\Delta \varphi / 2)}{\Delta \varphi \Delta \lambda}, \\
& \sum_{i=1}^{n}\left(x_{i}^{2}+y_{i}^{2}\right)=\frac{n R^{2}}{\Delta \varphi \Delta \lambda} \int_{\varphi_{a}}^{\varphi_{b}} \int_{\lambda_{a}}^{\lambda_{b}} \cos { }^{2} \varphi d \lambda d \varphi \\
& =\frac{n R^{2}}{2 \Delta \varphi}\left(\Delta \varphi+\frac{\sin 2 \varphi_{b}-\sin 2 \varphi_{a}}{2}\right) \\
& =\frac{n R^{2}}{2 \Delta \varphi}\left(\Delta \varphi+\cos 2 \varphi_{m} \sin \Delta \varphi\right), \\
& \sum_{i=1}^{n}\left(x_{i}^{2}+z_{i}^{2}\right)=\frac{n R^{2}}{\Delta \varphi \Delta \lambda}
\end{aligned}
$$

$$
\begin{aligned}
& \times \int_{\varphi_{a}}^{\varphi_{b}} \int_{\lambda_{a}}^{\lambda_{b}}\left(\cos ^{2} \varphi \cos ^{2} \lambda+\sin ^{2} \varphi\right) d \lambda d \varphi \\
& =\frac{n R^{2}}{\Delta \varphi \Delta \lambda}\left\{\frac{1}{4}\left(\Delta \lambda+\frac{\sin 2 \lambda_{b}-\sin 2 \lambda_{a}}{2}\right)\right. \\
& \times\left(\Delta \varphi+\frac{\sin 2 \varphi_{b}-\sin 2 \varphi_{a}}{2}\right) \\
& \left.+\frac{\Delta \lambda}{2}\left(\Delta \varphi+\frac{\sin 2 \varphi_{b}-\sin 2 \varphi_{a}}{2}\right)\right\} \\
& =\frac{n R^{2}}{\Delta \varphi \Delta \lambda}\left\{\frac{1}{4}\left(\Delta \lambda+\cos 2 \lambda_{m} \sin \Delta \lambda\right)\right. \\
& \times\left(\Delta \varphi+\cos 2 \varphi_{m} \sin \Delta \varphi\right) \\
& \left.+\frac{\Delta \lambda}{2}\left(\Delta \varphi-\cos 2 \varphi_{m} \sin \Delta \varphi\right)\right\}, \\
& \sum_{i=1}^{n}\left(y_{i}^{2}+z_{i}^{2}\right)=\frac{n R^{2}}{\Delta \varphi \Delta \lambda} \\
& \times \int_{\varphi_{a}}^{\varphi_{b}} \int_{\lambda_{a}}^{\lambda_{b}}\left(\cos ^{2} \varphi \sin ^{2} \lambda+\sin ^{2} \varphi\right) d \lambda d \varphi \\
& =\frac{n R^{2}}{\Delta \varphi \Delta \lambda}\left\{\frac{1}{4}\left(\Delta \lambda-\frac{\sin 2 \lambda_{b}-\sin 2 \lambda_{a}}{2}\right)\right. \\
& \times\left(\Delta \varphi+\frac{\sin 2 \varphi_{b}-\sin 2 \varphi_{a}}{2}\right) \\
& \left.+\frac{\Delta \lambda}{2}\left(\Delta \varphi+\frac{\sin 2 \varphi_{b}-\sin 2 \varphi_{a}}{2}\right)\right\} \\
& =\frac{n R^{2}}{\Delta \varphi \Delta \lambda}\left\{\frac{1}{4}\left(\Delta \lambda-\cos 2 \lambda_{m} \sin \Delta \lambda\right)\right. \\
& \times\left(\Delta \varphi+\cos 2 \varphi_{m} \sin \Delta \varphi\right) \\
& \left.+\frac{\Delta \lambda}{2}\left(\Delta \varphi-\cos 2 \varphi_{m} \sin \Delta \varphi\right)\right\} \text {. }
\end{aligned}
$$

Consequently, the Tykhonov matrix in Eq. (7) can be obtained with respect to an ideal network configuration through Eqs. (12)-(17). The regularization constant is usually obtained through an ad-hoc method (Tarantola, 2005). Various methods such as the L-Curve, generalized cross-validation, maximum likelihood, Morozov's discrepancy principle, quasi-optimality criterion, and the Cp-Plot, have been developed in different contexts (Mallows, 1973; Hansen, 1992; Golub and von Matt, 1997). However, the success of each method to determine the regularization constant depends on the specific application (Hoerl and Kennard, 1970). One suitable method for one specific problem could have an over-smoothing or under-smoothing effect on another. In general, all the methods employ the variation of the Weighted Residuals Sum of Squares (WRSS) with respect to the regularization constant. The chosen constant should balance the WRSS and the constraints. The empirical determination of the regularization constant from WRSS plots is also very common and has been shown to be a sufficient method in many geophysical problems (Bürgmann et al., 2002; Wright et al., 2003; Aktuğ et al., 2010).

Equation (7) can also be interpreted from a Bayesian point of view, where a priori constraints are applied over the parameters through the Tykhonov matrix. Since the Tykhonov matrix was derived by using an ideal network distribution, the covariance constructed upon such an ideal 


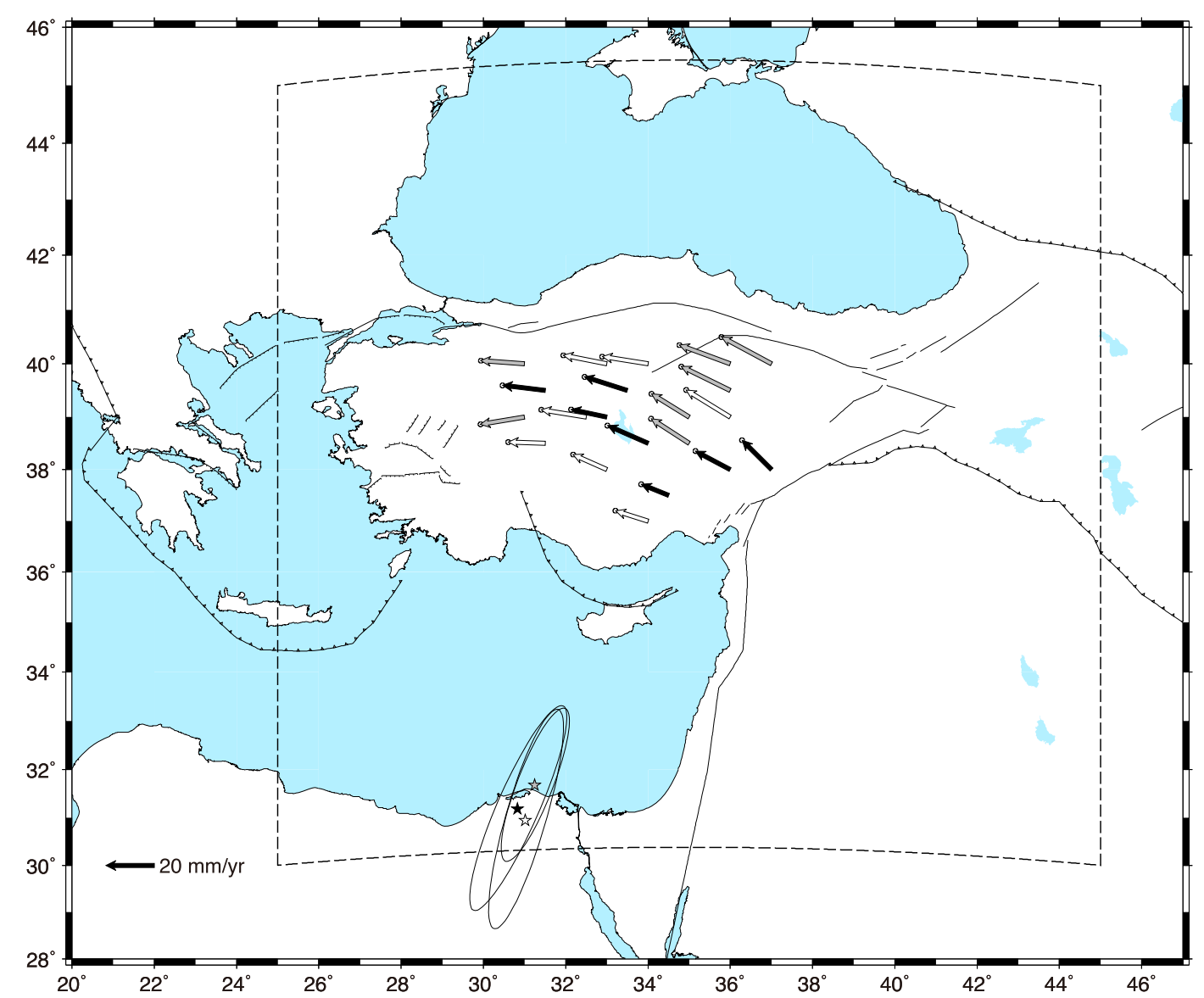

Fig. 1. Three synthetic networks (net \#1 = black, net \#2 = white, net \#3 = gray arrows) and the estimated positions of Euler poles. The ellipses for velocities and the Euler poles are at $95 \%$ confidence level. The area with dashed borders represents the borders of an ideal regional network distribution.

distribution is too perfect. Such a non-realistic covariance matrix has also too optimistic error estimates. Using such a covariance matrix corresponds to putting too tight constraints on the a priori parameters. However, the a priori values of the Euler parameters are obtained from many different observations of varying accuracy. Therefore, the $a$ priori values are generally less accurate than implied by the covariance matrix of a perfect network distribution. To be able to use less accurate a priori constraints while preserving the intrinsic correlations of constraints, the cofactor matrix obtained in Eq. (5) can be transformed into a correlation matrix and a new inverse cofactor matrix with loose constraints can be formed as:

$$
\begin{aligned}
& \mathbf{E}=\mathbf{D}^{-1 / 2} \Sigma_{\hat{\xi}} \mathbf{D}^{-1 / 2}, \\
& \Gamma^{T} \boldsymbol{\Gamma}=\left(\mathbf{C}_{x_{0}}^{1 / 2} \mathbf{E} \mathbf{C}_{x_{0}}^{1 / 2}\right)^{-1},
\end{aligned}
$$

where $\mathbf{E}$ is the correlation matrix, $\mathbf{D}$ is the diagonal matrix formed by using the computed covariance as $\mathbf{D}=\operatorname{diag}\left(\Sigma_{\hat{\xi}}\right)$, $\mathbf{C}_{x_{0}}$ is the diagonal matrix of loose a priori constraints for $a$ priori values of the parameters $\left(x_{0}\right)$. $\mathbf{C}_{x_{0}}$ is constructed by putting the variances of the parameters in the diagonals.

\section{Numerical Application}

To demonstrate the efficiency of the regularized estimation of Euler vector components, three synthetic velocity fields were formed using a pre-defined Euler vector. The
Table 1. True and a priori Euler pole parameters.

\begin{tabular}{lccc}
\hline & $\varphi_{p}$ & $\lambda_{p}$ & $\omega$ \\
& $\left({ }^{\circ}\right)$ & $\left({ }^{\circ}\right)$ & $(\% / \mathrm{Myr})$ \\
\hline True & 32.0000 & 32.0000 & 1.4000 \\
A priori & 30.0000 & 31.0000 & 2.0000 \\
\hline
\end{tabular}

Table 2. Estimated Euler pole parameters with standard least squares.

\begin{tabular}{ccccc}
\hline Net \# & $\begin{array}{c}\varphi_{p} \\
\left({ }^{\circ}\right)\end{array}$ & $\begin{array}{c}\lambda_{p} \\
\left({ }^{\circ}\right)\end{array}$ & $\begin{array}{c}\omega \\
\left({ }^{\circ} / \mathrm{Myr}\right)\end{array}$ & $\begin{array}{c}\text { MSE } \\
\left({ }^{\circ} / \mathrm{Myr}\right)\end{array}$ \\
\hline 1 & 31.1836 & 30.8270 & 1.0812 & 0.3200 \\
2 & 30.9492 & 31.0188 & 1.1277 & 0.2739 \\
3 & 31.6771 & 31.2491 & 1.2739 & 0.1272 \\
\hline
\end{tabular}

three different velocity field configurations are shown in Fig. 1. Since the relative performance of the regularized estimation with respect to the standard least squares will be shown in terms of MSE, it is necessary to use synthetic networks with the known Euler vector components. The known Euler parameters and the a priori values used in the estimation are given in Table 1. Each velocity field of the three networks was obtained using the same set of Euler parameters given in Table 1, and random noise sampled from a normal distribution with a standard deviation of $\pm 1 \mathrm{~mm} / \mathrm{yr}$ was then added to the velocity components, which is typi- 


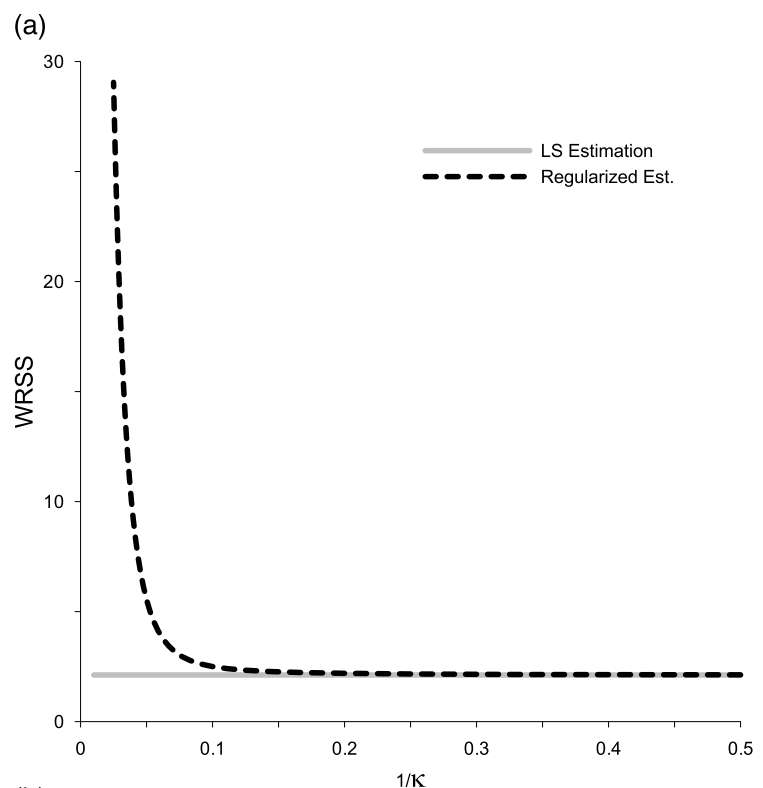

(b)

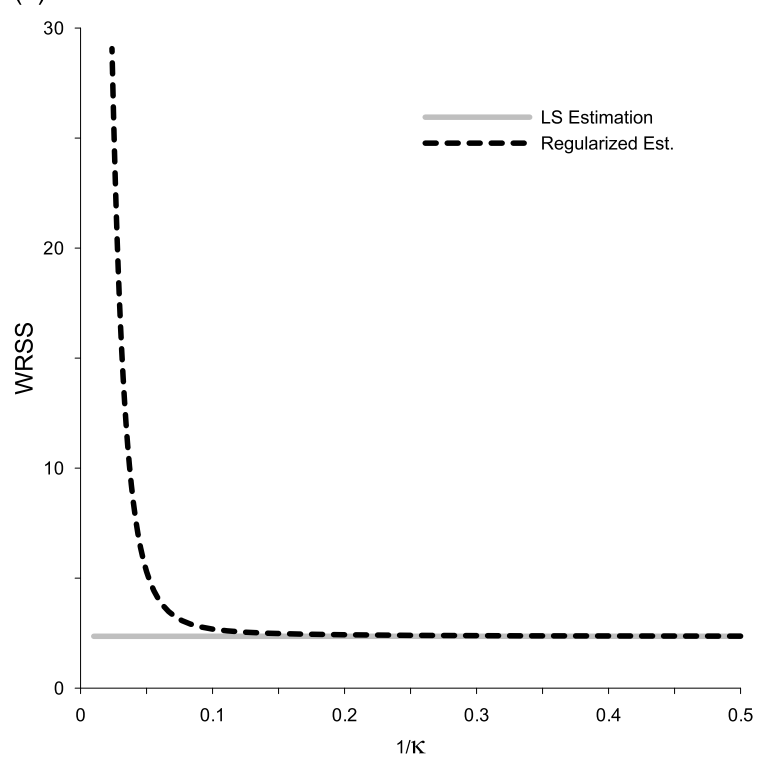

(c)

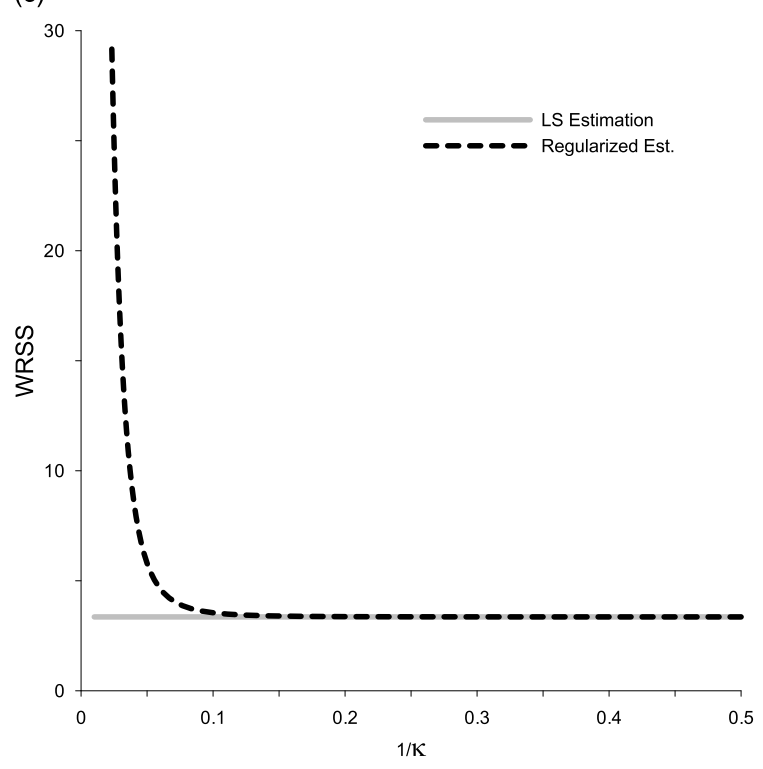

Fig. 2. Weighted residual sum of the squares as a function of regularization constant (a) net \#1 (b) net \#2 (c) net \#3.

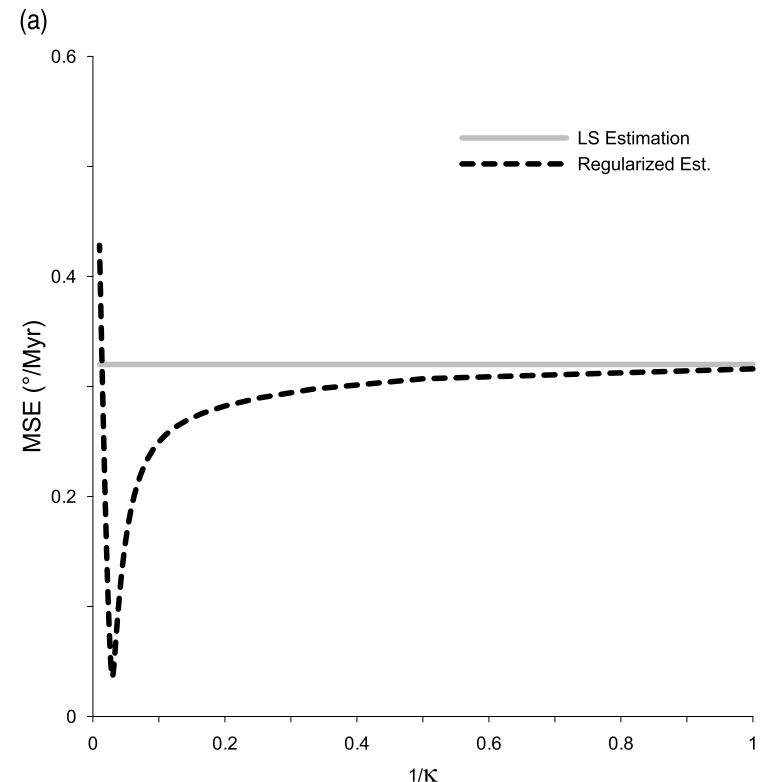

(b)
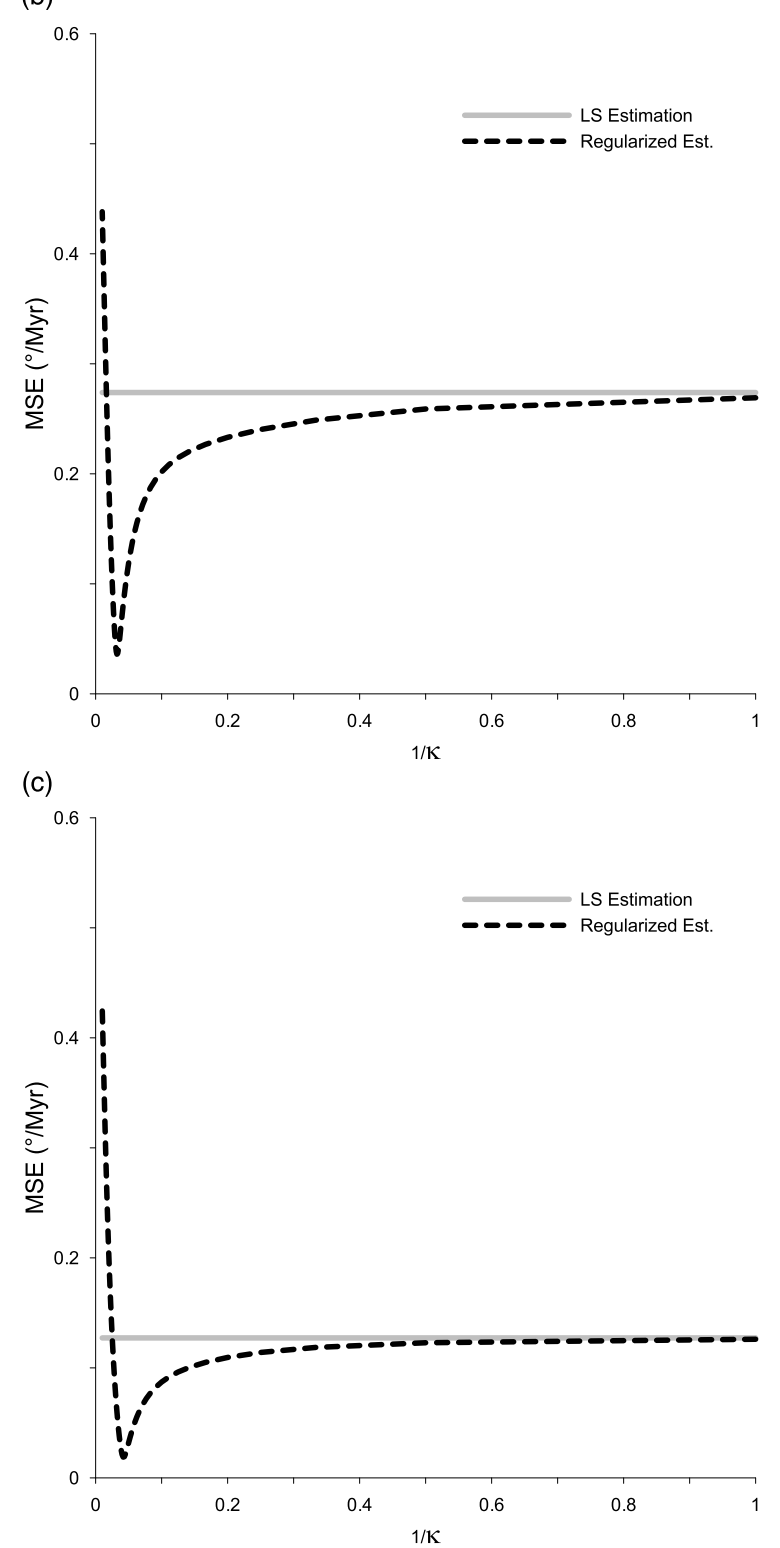

Fig. 3. Mean squared error as a function of regularization constant (a) net \#1 (b) net \#2 (c) net \#3. 
Table 3. Estimated Euler pole parameters with regularized method.

\begin{tabular}{ccccc}
\hline Net \# & $\begin{array}{c}\varphi_{p} \\
\left({ }^{\circ}\right)\end{array}$ & $\begin{array}{c}\lambda_{p} \\
\left(^{\circ}\right)\end{array}$ & $\begin{array}{c}\omega \\
\left({ }^{\circ} / \mathrm{Myr}\right)\end{array}$ & $\begin{array}{c}\text { MSE } \\
\left({ }^{\circ} / \mathrm{Myr}\right)\end{array}$ \\
\hline 1 & 31.9541 & 31.2776 & 1.2550 & 0.1457 \\
2 & 31.7478 & 31.3165 & 1.2982 & 0.1029 \\
3 & 31.7042 & 31.3392 & 1.3141 & 0.0872 \\
\hline
\end{tabular}

Table 4. MSE values with different $a$ priori values.

\begin{tabular}{|c|c|c|c|c|c|}
\hline Net \# & $\begin{array}{l}\varphi_{p} \\
\left(^{\circ}\right)\end{array}$ & $\begin{array}{l}\lambda_{p} \\
\left(^{\circ}\right)\end{array}$ & $\begin{array}{c}\omega \\
(/ \mathrm{Myr})\end{array}$ & $1 / \lambda$ & $\begin{array}{c}\text { MSE } \\
(\% / \mathrm{Myr})\end{array}$ \\
\hline 1 & 33 & 33 & 1 & 0.1 & 0.2226 \\
\hline 1 & 33 & 33 & 2 & 0.1 & 0.2036 \\
\hline 1 & 33 & 31 & 1 & 0.1 & 0.2443 \\
\hline 1 & 33 & 31 & 2 & 0.1 & 0.1677 \\
\hline 1 & 31 & 33 & 1 & 0.1 & 0.3473 \\
\hline 1 & 31 & 33 & 2 & 0.2 & 0.1394 \\
\hline 1 & 31 & 31 & 1 & 0.1 & 0.3556 \\
\hline 1 & 31 & 31 & 2 & 0.2 & 0.1461 \\
\hline 2 & 33 & 33 & 1 & 0.1 & 0.1808 \\
\hline 2 & 33 & 33 & 2 & 0.1 & 0.2482 \\
\hline 2 & 33 & 31 & 1 & 0.1 & 0.1939 \\
\hline 2 & 33 & 31 & 2 & 0.1 & 0.2286 \\
\hline 2 & 31 & 33 & 1 & 0.1 & 0.3085 \\
\hline 2 & 31 & 33 & 2 & 0.2 & 0.1273 \\
\hline 2 & 31 & 31 & 1 & 0.1 & 0.3116 \\
\hline 2 & 31 & 31 & 2 & 0.2 & 0.1283 \\
\hline 3 & 33 & 33 & 1 & 0.1 & 0.1143 \\
\hline 3 & 33 & 33 & 2 & 0.2 & 0.0641 \\
\hline 3 & 33 & 31 & 1 & 0.2 & 0.1298 \\
\hline 3 & 33 & 31 & 2 & 0.2 & 0.0502 \\
\hline 3 & 31 & 33 & 1 & 0.2 & 0.1956 \\
\hline 3 & 31 & 33 & 2 & 0.2 & 0.0430 \\
\hline 3 & 31 & 31 & 1 & 0.2 & 0.2029 \\
\hline 3 & 31 & 31 & 2 & 0.2 & 0.0505 \\
\hline
\end{tabular}

cal for GNSS observations (Aktuğ et al., 2009b). To sufficiently account for the non-rigid behavior of the sites, as well as the possible unmodelled observation errors, a nonrigid deformation of 1-2 mm/yr was also randomly added to the velocities. Such non-rigid behavior is very common in GNNS networks and usually observed in residual velocities with respect to a plate-fixed reference frame (McClusky et al., 2000; Nocquet et al., 2001). The velocities shown in Fig. 1 include the added noise.

The standard least-squares solutions for the three networks are given in Table 2. As is clear in Table 2, the Euler vector is highly sensitive to the geometry of the sites. The magnitude of rotation varies up to $0.3^{\circ} / \mathrm{Myr}$, which makes it difficult to compare the results with the results of the studies. In the regularized estimation, the Tykhonov matrix was constructed with loose constraints of $2^{\circ}, 2^{\circ}$ and $1^{\circ} / \mathrm{Myr}$ for the latitude, longitude and the rotation rate, respectively. Such very loose constraints are compatible with the a priori values which were deliberately chosen to be imprecise. The regularization constants were determined empirically as 0.10 for all three networks using the WRSS plots of the networks given in Fig. 2, similar to that given in Aktuğ et al. (2010). The necessary geographical bounds for construct- ing the Tykhonov matrix were chosen as shown in Fig. 1 by a dashed line. Finally, the Mean Squared errors of both least squares and the proposed method was computed as:

$$
\operatorname{tr}(\operatorname{MSE}(\hat{\mathbf{x}}))=\operatorname{tr}\left(E\left\{(\mathbf{x}-\hat{\mathbf{x}})(\mathbf{x}-\hat{\mathbf{x}})^{T}\right\}\right)
$$

where $E\{\cdot\}, \mathbf{x}$ and $\hat{\mathbf{x}}$ are the expectation operatör, the true values vector, and the estimated values vector of the parameters, respectively. The results of regularized estimation are given in Table 3. The MSE plots of both standard least squares and regularized estimation are given in Fig. 3.

To investigate the effect of different $a$ priori values, the numerical study was repeated with different sets of $a$ priori values. The regularization constant was chosen separately for each set. The different sets of a priori values, and the MSE of each trial, are given in Table 4.

\section{Results and Conclusion}

The Euler vector has been an indispensible tool for modeling plate tectonics, as well as for approximating the rigid motion of sites. However, the number of sites located in the rigid parts of the plates is often limited. Such a limitation is often coupled with uncertainty about the rigid behavior of a site represented in the residuals of the velocities defined in plate-fixed frames. Considering that the estimation model of Euler vectors is in an Earth-centered Cartesian frame, the velocity vectors on a small plate provides a limited geographic coverage, presenting a multicollinear problem.

It has been shown that a very similar network configuration can make a huge difference in the estimated parameters. For many regional networks, either the latitude or the longitude of the Euler pole is nearly collinear with the magnitude of the rotation rate, such that an iterative solution does not converge in the direct estimation of the Euler pole parameters. In the given example, the latitude of the Euler pole is highly correlated with the rotation rate since the Euler pole is almost in the south of the Anatolian plate. The normal equation matrix is a function of the geometry of the distribution of the network, and the condition number of the normal equation matrix is an indication of how well-posed the problem is. Ill-posed problems are generally identified by the high condition numbers of the normal equation matrix (Hansen, 2010). For instance in the given examples, while the condition number of the normal equation matrix in a direct estimation of the Euler pole parameters is about $\sim 10^{14}$, it decreases to $\sim 10^{3}$ in estimating the Cartesian Euler vector, which is still very large and poses a weakly multicollinear problem. The contribution of the proposed method depends on the quality of the network distribution and the $a$ priori values. For a nearly ideal network, the contribution could be negligible. The distribution of errors for the sites, and the geometry of the sites, determine the performance of the proposed method. The performance of the proposed method, when using different a priori values, is also dependent on the network geometry. The same a priori values used in three networks affected the performance differently due to the different network configurations and error distribution. However, using the regularization constant, the proposed method can always be tuned to give better MSE, or at least, the same MSE with the standard least-squares. 
The smaller is the regularization constant, the closer will be the estimate to the standard least-squares. Regardless of the MSE performance, the proposed method also provides a homogenous framework to compare the results of different studies.

Acknowledgments. The authors would like to thank the Editor and two anonymous reviewers for their valuable comments and suggestions to improve the manuscript. All the figures were prepared using the software of Wessel and Smith (1995).

\section{References}

Aktuğ, B., Weakly Multicollinear Datum Transformations, J. Surv. Eng., 138(4), 184-192, 2012.

Aktuğ, B., O. Lenk, M. A. Gürdal, and A. Kilicoglu, Establishment of regional reference frames for detecting active deformation areas in Anatolia, Stud. Geophys. Geod., 2, 53, 169-183, 2009a.

Aktuğ, B., J. M. Nocquet, A. Cingoz, B. Parsons, Y. Erkan, P. England, O. Lenk, M. A. Gurdal, A. Kilicoglu, H. Akdeniz, and A. Tekgul, Deformation of western Turkey from a combination of permanent and campaign GPS data: Limits to block-like behavior, J. Geophys. Res., 114, B10404, 2009b.

Aktuğ, B., B. Kaypak, and R. N. Çelik, Source parameters of 03 February 2002 Çay Earthquake, Mw6.6 and aftershocks from GPS Data, Southwestern Turkey, J. Seismol., 14, 445-456, 2010.

Altamimi, Z., P. Sillard, and C. Boucher, ITRF2000: A new release of the International Terrestrial Reference Frame for earth science applications, J. Geophys. Res., 107(B10), 2114, doi:10.1029/2001JB000561, 2002.

Argus, D. F. and R. G. Gordon, No-net-rotation model of current plate velocities incorporating plate motion model NUVEL-1, Geophys. Res. Lett., 18, 2039-2042, 1991.

Bürgmann, R., M. A. Ayhan, E. J. Fielding, T. Wright, S. McClusky, B. Aktug, C. Demir, O. Lenk, and A. Türkezer, Deformation during the 12 November 1999, Düzce, Turkey Earthquake, from GPS and InSAR Data, Bull. Seismol. Soc. Am., 92, 161-171, 2002.

DeMets, C., R. G. Gordon, D. F. Argus, and S. Stein, Current plate motions, Geophys. J. Int., 101, 425-478, 1990.

DeMets, C., R. G. Gordon, D. F. Argus, and S. Stein, Effect of recent revisions to the geomagnetic reversal time scale on estimates of current plate motions, Geophys. Res. Lett., 21, 2191-2194, 1994.

DeMets, C., R. G. Gordon, and D. F. Argus, Geologically current plate motions, Geophys. J. Int., 181, 1-80, 2010.

Flores, A., G. Ruffini, and A. Rius, 4D tropospheric tomography using GPS slant wet delays, Ann. Geophys., 18, 223-234, 2000.

Golub, G. and U. von Matt, Tikhonov regularization for large scale problems, Stanford SCCM Report, 97-03, Stanford, California, 1997.

Gripp, A. E. and R. G. Gordon, Current plate velocities relative to the hotspots incorporating the NUVEL-1 global plate motion model, Geophys. Res. Lett., 17, 1109-1112, 1990.

Gripp, A. E. and R. G. Gordon, Young tracks of hotspots and current plate velocities, Geophys. J. Int., 150, 321-361, 2002.

Hansen, P. C., Analysis of discrete ill-posed problems by means of the L-curve, SIAM Rev., 34, 561-580, 1992.
Hansen, P. C., Discrete Inverse Problems: Insight and Algorithms, SIAM, Philadelphia, 2010

Hoerl, A. E. and R. W. Kennard, Ridge regression: Biased estimation for nonorthogonal problems, Technometrics, 12, 55-67, 1970.

Howe, B. M., K. Runciman, and J. A. Secan, Tomography of ionosphere: Four dimensional simulations, Radio Sci., 33(1), 109-128, 1998.

Kreemer, C., W. E. Holt, and A. J. Haines, An integrated global model of present-day plate motions and plate boundary deformation, Geophys. J. Int., 154, 8-34, 2003.

Mallows, C. L., Some comments on Cp, Technometrics, 15, 661-675, 1973.

McCaffrey, R., Estimates of modern arc-parallel strain rates in forearcs, Geology, 24, 27-30, 1996.

McCaffrey, R., Crustal block rotations and plate coupling, in Plate Boundary Zones, edited by S. Stein and J. Freymueller, AGU Geodynamics Series, 30, 101-122, 2002.

McClusky, S., S. Bassalanian, A. Barka, C. Demir, S. Ergintav, I. Georgiev, O. Gurkan, M. Hamburger, K. Hurst, H.-G. Hans-Gert, K. Karstens, G. Kekelidze, R. King, V. Kotzev, O. Lenk, S. Mahmoud, A. Mishin, M. Nadariya, A. Ouzounis, D. Paradissis, Y. Peter, M. Prilepin, R. Relinger, I. Sanli, H. Seeger, A. Tealeb, M. N. Toksaz, and G. Veis, Global Positioning system constraints on plate kinematics and dynamics in the eastern Mediterranean and Caucasus, J. Geophys. Res., 105(B3), 5695-5719, 2000.

Meade, B. J. and B. H. Hager, Block models of crustal motion in southern California constrained by GPS measurements, J. Geophys. Res., 110, B03403, 2005.

Nocquet, J. M., E. Calais, Z. Altamimi, P. Sillard, and C. Boucher, Intraplate deformation in western Europe deduced from an analysis of the International Terrestrial Reference Frame 1997 (ITRF97) velocity field, J. Geophys. Res., 106(B6), 11239-11257, 2001.

Phillips, D. L., A technique for the numerical solution of certain integral equations of the first kind, J. Assoc. Comput. Mach., 9, 84-96, 1962.

Prawirodirdjo, L. and Y. Bock, Instantaneous global plate motion model from 12 years of continuous GPS observations, J. Geophys. Res., 109, B08405, 2004.

Qiang, Z., Z. Wenyao, and X. Yongqin, Global plate motion models incorporating the velocity field of ITRF96, Geophys. Res. Lett., 26(18), 2813-2816, 1999.

Sella, G., T. Dixon, and A. Mao, REVEL: A model for recent plate velocities from space geodesy, J. Geophys. Res., 107(B4), 2002.

Tarantola, P., Inverse Problem Theory, SIAM, Philadelphia, 2005.

Tykhonov, A. N., The regularization of incorrectly posed problems, Soviet Math. Doklady, 4, 1624-1627, 1963.

Wessel, P. and W. H. F. Smith, New version of the generic mapping tools released, Eos Trans. AGU, 76(329), 1995.

Wright, T. J., Z. Lu, and C. Wicks, Source model for the Mw 6.7, 23 October 2002, Nenana Mountain Earthquake (Alaska) from InSAR, Geophys. Res. Lett., 30(18), 2003.

B. Aktuğ (e-mail: bahadir.aktug@boun.edu.tr) and Ö. Yıldırım 